\title{
Randomized RANSAC with Sequential Probability Ratio Test
}

\author{
Jiří Matas and Ondřej Chum \\ Center for Machine Perception, Dept. of Cybernetics, CTU Prague, Karlovo nám 13, CZ 12135 \\ [chum, matas] ¿cmp.felk.cvut.cz
}

\begin{abstract}
A randomized model verification strategy for RANSAC is presented. The proposed method finds, like RANSAC, a solution that is optimal with user-controllable probability $\eta$. A provably optimal model verification strategy is designed for the situation when the contamination of data by outliers is known, i.e. the algorithm is the fastest possible (on average) of all randomized RANSAC algorithms guaranteeing $1-\eta$ confidence in the solution. The derivation of the optimality property is based on Wald's theory of sequential decision making. The R-RANSAC with SPRT, which does not require the a priori knowledge of the fraction of outliers and has results close to the optimal strategy, is introduced. We show experimentally that on standard test data the method is 2 to 10 times faster than the standard RANSAC and up to 4 times faster than previously published methods.
\end{abstract}

\section{Introduction}

The $^{1}$ RANSAC (RANdom SAmple Consensus) algorithm introduced by Fishler and Bolles in 1981 [3] is a widely used robust estimator that has become a de facto standard in the field of computer vision [4]. RANSAC and related hypothesize-and-verify methods $[11,10,9,7,1,8]$ have been applied in many vision problems.

The RANSAC algorithm proceeds as follows. Repeatedly, subsets of the input data (e.g. a set of tentative correspondences) are randomly selected and model parameters fitting the sample are computed. In a second step, the quality of the parameters is evaluated on the input data. Different cost functions have been proposed [10], the standard being the number of inliers, i.e. the number of data points consistent with the model. The process is terminated when the probability of finding a better model becomes lower than a usercontrolled probability $\eta$. The $1-\eta$ confidence in the solution holds for all levels of contamination of the input data, i.e. for any number of outliers within the input data.

\footnotetext{
${ }^{1}$ The authors were supported by the Czech Ministry of Education project 1M6840770004 and by the EU Commission project IST-004176 COSPAL.
}

The speed of standard RANSAC depends on two factors. The percentage of outliers determines the number of random samples needed to guarantee the $1-\eta$ confidence in the solution. The time needed to assess the quality of a hypothesized model parameters is proportional to the number $N$ of the input data points. Almost all models whose quality is verified are incorrect with arbitrary parameters originating from contaminated samples. Such models are consistent with only a small number of the data points. In [6], Matas and Chum showed how this property can be exploited to increase the speed of RANSAC. The algorithm, called R-RANSAC, speeds up the model evaluation step by introducing a two-stage procedure. First, a statistical test is performed on $d$ randomly selected data points $(d \ll N)$. Evaluation of the remaining $N-d$ data points is carried out only if the first $d$ data points are inliers. The speed up of the R-RANSAC depends on the probabilities of the two types of errors committed in the pre-test, the rejection of an uncontaminated model and the acceptance of a contaminated model. The idea was modified by Nistér to include competitive verification of models and exploited in an impressive real-time structure from motion system [8]. The main limitation of Nistér's pre-emptive RANSAC is that a fixed number of models is evaluated, which is equivalent to an a priori assumption that the fraction of inliers is known. This limits the applicability of pre-emptive RANSAC in some problems where the fraction of inliers ranges widely, such as in wide baseline stereo.

As noted in [6], the two-stage procedure of R-RANSAC is not optimal. As a main contribution of this paper, we define an optimal hypothesis evaluation procedure, i.e. a method for randomized model quality evaluation that returns, in the fastest average time possible, a solution with the confidence $1-\eta$. The derivation of the optimality property is based on Wald's theory of sequential decision making [12].

From the statistical point of view, the evaluation of the model quality can be formulated as a sequential test as follows. The hypothesis generation step proposes a model. It is either 'good', i.e. it leads to the optimal solution (the solution with maximal support), or it is 'bad', i.e. one or more of the data points in the sample are an outliers. The property 
'good' is a hidden state that is not directly observable but is statistically linked to observable events. The observable events are "a data point (correspondence) is/is-not consistent with the model". In sequential testing, as applied e.g. in industrial inspection, the problem is to decide whether the model (or the batch of products) is 'good' or 'bad' in the shortest possible time (i.e. making the smallest number of observations) and yet satisfying the predefined bounds on the probabilities of the two possible errors - accepting a 'bad' model as 'good' and vice versa. Wald proposed the sequential probability ratio test (SPRT) and showed [12] that, given errors bound on the errors of the first and second kind, it minimizes the the number of observations (time to decision $)^{2}$.

Wald's SPRT test is a solution of a constrained optimization problem. The user supplies the acceptable probabilities of the errors of the first and the second kind and the resulting optimal test is a trade-off between time to decision (or the cost of observations) and the errors committed. However, when evaluating RANSAC, the situation is different. First of all, a 'good' model is always evaluated for all data points (correspondences) since the number of inliers is one of the outputs of the algorithms. So the only error that can be committed is an early rejection of a 'good' model (error of the first kind). But this only means that more samples have to be drawn to achieve the required confidence $1-\eta$ of finding the optimal solution. So unlike in the classical setting, we are solving a global optimization problem, minimizing a single real number - the time to decision, since the consequence of an error is also a loss of time.

The structure of this paper is as follows. First, in Section 2, we introduce the relevant parts of Wald's decision theory and show how its results can be brought to bear on the problem of minimizing RANSAC run time. The RANSAC with SPRT algorithm is described in detail in Section 3. In Section 4, the theoretical results are experimentally verified on standard stereo matching problems. The paper is concluded in Section 5.

\section{The Optimal Sequential Test}

The model evaluation step of the optimal R-RANSAC proceeds as Wald's sequential probability ratio test (SPRT) with the probability $\alpha$ of rejecting a 'good' sample set to maximize the speed of the whole RANSAC process. To understand the operation of R-RANSAC with SPRT, some familiarity with Wald's decision theory is required. We therefore introduce its relevant parts. Some of the results are presented in a form that is not fully general, but sufficient for the derivation of the R-RANSAC with SPRT algorithm.

\footnotetext{
${ }^{2}$ Precisely speaking, the SPRT is only approximately optimal. However, the approximation has been shown by Wald to be so close to the optimum that it is considered the optimal test for all practical purposes.
}

Some of Wald's terminology is modified in order to make the exposition more accessible.

In the model evaluation step, our objective is to decide between the hypothesis $H_{g}$ that model is 'good' and the alternative hypothesis $H_{b}$ that the model is 'bad'. A 'good' model is computed from an all-inlier sample. The Wald's SPRT is based on the likelihood ratio [12]

$$
\lambda_{j}=\prod_{r=1}^{j} \frac{p\left(x_{r} \mid H_{b}\right)}{p\left(x_{r} \mid H_{g}\right)}=\lambda_{j-1} \cdot \frac{p\left(x_{j} \mid H_{b}\right)}{p\left(x_{j} \mid H_{g}\right)},
$$

a ratio of two conditional probabilities of an observation $x_{r}$ under the assumptions of $H_{g}$ and $H_{b}$ respectively. In RANSAC, $x_{r}$ is equal to 1 if the $r$-th data point is consistent with a model with parameters $\theta$ and 0 otherwise ${ }^{3}$. For example, a correspondence is consistent with (i.e. supporting) an epipolar geometry represented by a fundamental matrix F if its Sampson's error is smaller than some predefined threshold [4]. The probability $p\left(1 \mid H_{g}\right)$ that any randomly chosen data point is consistent with a 'good' model is approximated by the fraction of inliers $\varepsilon$ among the data points ${ }^{4}$. The probability of a data point being consistent with a 'bad' model is modeled as a probability of a random event with Bernoulli distribution with parameter $\delta$ : $p\left(1 \mid H_{b}\right)=\delta$. The process of estimation of $\delta$ and $\varepsilon$ is discussed in Section 3.

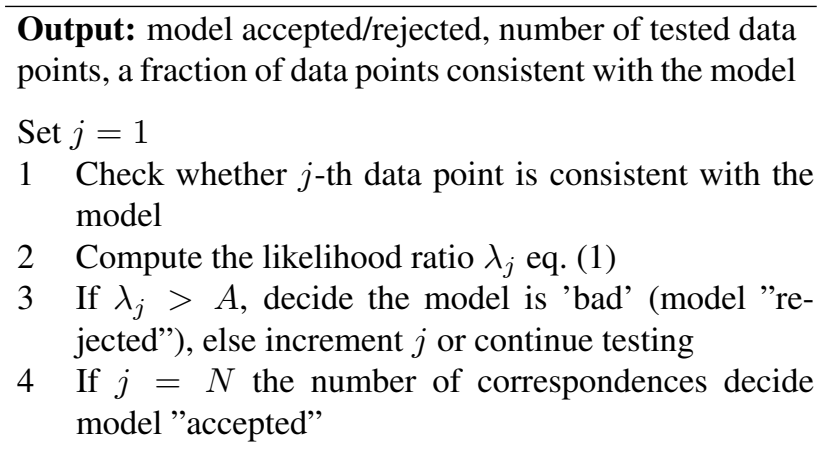

Algorithm 1: The adapted sequential probability ratio test (Adapted SPRT).

After each observation the standard Wald's SPRT makes one of three decisions: accept a 'good' model, reject a 'bad' model, or continue testing. Since in RANSAC the total number of inliers is needed to decide on termination, nothing is gained by an early decision in favor of a 'good' model. Therefore the option of an early acceptance of the model

\footnotetext{
${ }^{3}$ In this paper, we address the case of the step cost function only. The method can be extended to a smooth cost function as used in MLES AC [10], for details see [2].

${ }^{4}$ The probability $p\left(1 \mid H_{g}\right)$ would be exactly $\varepsilon$ if the data points were selected with replacement. Since the objective of the verification is to count the size of the support of the model, the correspondences are drawn without replacement.
} 
has been removed in the adapted SPRT (Alg. 1). The full SPRT is described e.g. in Wald [12] and, in a more accessible form, in Lee [5].

\subsection{The optimal value of the decision threshold}

The decision threshold $A$ is the only parameter of the Adapted SPRT. We show how to set it to achieve optimal performance, i.e. minimal average RANSAC running time given the probabilities $\delta$ and $\varepsilon$. We use the following theorems (for proofs, see [12]).

Theorem 1 The probability $\alpha$ of rejecting a 'good' model in SPRT $\alpha \leq 1 / A$.

Proof: Wald's theorem [12, p. 41] states $\alpha \leq(1-\beta) / A$, where $\beta$ stands for the probability that a 'bad' model is incorrectly accepted as 'good'. In the adapted SPRT, since the only decision of the test can be "reject", $\beta=0$ and thus $\alpha \leq 1 / A$.

The approximation $\alpha \approx 1 / A$ is close and is often used.

Theorem 2 (Wald's lemma) The average number of observations (checked data points) carried out while testing a 'bad' model is $C^{-1} \log A$, where

$$
C=p\left(0 \mid H_{b}\right) \log \frac{p\left(0 \mid H_{b}\right)}{p\left(0 \mid H_{g}\right)}+p\left(1 \mid H_{b}\right) \log \frac{p\left(1 \mid H_{b}\right)}{p\left(1 \mid H_{g}\right)}
$$

Proof: According to [12, p. 53]

$$
C=E\left(\log \frac{p\left(x \mid H_{b}\right)}{p\left(x \mid H_{g}\right)}\right) .
$$

The value of $x$ is from $\{0,1\}$. The expectation $E$ is a sum of two terms weighted by probability $p\left(x \mid H_{b}\right)$. Equation (2) follows.

In the particular case of RANSAC, $p\left(1 \mid H_{b}\right)=\delta$, $p\left(0 \mid H_{b}\right)=1-\delta, p\left(0 \mid H_{g}\right)=1-\varepsilon$, and $p\left(1 \mid H_{g}\right)=\varepsilon$. Therefore the average number of verified correspondences per model is:

$$
\frac{\log A}{C}=\left((1-\delta) \log \frac{1-\delta}{1-\varepsilon}+\delta \log \frac{\delta}{\varepsilon}\right)^{-1} \log A .
$$

The value of $A$ influences the total running time in two opposing ways. The larger the value of $A$, the smaller the probability of rejection of a 'good' model. On the other hand, the number of correspondendes verified per model increases with $\log A$ (eq (4)). We wish to set $A$ to achieve minimal average time needed to find the solution.

The average time-to-solution in R-RANSAC is $t=\bar{k} \bar{t}_{s}$, where $\bar{k}$ is the average number of samples drawn until a 'good' model and $\bar{t}_{s}$ is the average testing time per sample. In the following, the time unit will be the time needed to check one data point. The probability $P_{g}$ of drawing a 'good' model is $P_{g}=\varepsilon^{m}$, where $m$ is the number of data points in the RANSAC sample. The number of tested samples before a 'good' one is drawn and not rejected is a random variable with geometric distribution and mean $\bar{k}=1 /\left(P_{g}(1-\alpha)\right) \approx 1 /\left(P_{g}(1-1 / A)\right)$. The average time $\bar{t}_{s}$ of processing a sample consists of two components: time $t_{M}$ needed to instantiate a model hypotheses given a sam$\mathrm{ple}^{5}$, and the average time of testing each hypothesis. Let $\bar{m}_{S}$ be the number of models that are verified per sample ${ }^{6}$ and $C^{-1} \log A$ be the average length of the SPRT (Theorem 2). The average time to the solution expressed as a function of $A$ is

$$
t(A)=\frac{1}{P_{g}(1-1 / A)}\left(t_{M}+\bar{m}_{S} \frac{\log A}{C}\right) .
$$

The formula (5) can be simplified to $t(A)=\frac{K_{1}+K_{2} \log A}{1-1 / A}$, where $K_{1}=t_{M} / P_{g}$ and $K_{2}=\bar{m}_{S} /\left(P_{g} C\right)$. We are interested in the optimal value of $\mathrm{A}$, i.e.

$$
A^{*}=\arg \min _{A} t(A) .
$$

The minimum is found by solving

$$
\frac{d t}{d A}=-\frac{K_{1}+K_{2}-K_{2} A+K_{2} \log A}{(A-1)^{2}}=0 .
$$

After rearrangements, we have

$$
A^{*}=\frac{K_{1}}{K_{2}}+1+\log A^{*}=\frac{t_{M} C}{\bar{m}_{S}}+1+\log A^{*} .
$$

Equation (6) has two real solutions for positive $K_{1} / K_{2}$, $A_{1}^{*}<1<A_{2}^{*}$. Since $\delta<\varepsilon$, the contribution to the likelihood ratio (eq. (1)) of a correspondence that is not consistent with the model is greater than 1 , therefore the solution of interest is $A^{*}>1$. This solution can be obtained as $A^{*}=\lim _{n \rightarrow \infty} A_{n}$, where $A_{0}=K_{1} / K_{2}+1$ and $A_{n+1}=K_{1} / K_{2}+1+\log \left(A_{n}\right)$. The series converges fast, typically within four iterations.

\section{R-RANSAC with SPRT}

The R-RANSAC with SPRT algorithm is outlined in Alg. 2. To fully specify the details of the algorithm, two issues have to be addressed. First, the estimation of parameters $\delta$ and $\varepsilon$; second, the termination criterion guaranteeing $1-\eta$ confidence in the solution has to be derived.

\footnotetext{
${ }^{5}$ Computing model parameters from a sample takes the same time as verification of $t_{M}$ data points.

${ }^{6}$ In the 7-pt algorithm for epipolar geometry estimation, 1 to 3 models have to be verified.
} 
Initialize $\varepsilon_{0}, \delta_{0}$, calculate $A_{0}$ and set $i=0$.

Repeat until the probability $\eta$ (eq. (10)) of finding a model with support larger than $\hat{\varepsilon}$ falls under a user defined confidence value $\eta_{0}$ :

\section{Hypothesis generation}

- Select a random sample of minimum size $m$ from the set of data points.

- Estimate model parameters $\theta$ fitting the sample.

\section{Verification}

Execute the SPRT (Alg. 1) and update the estimates if

a Model rejected: re-estimate $\delta$. If the estimate $\hat{\delta}$ differs from $\delta_{i}$ by more than $5 \%$ design $(i+1)$-th test $\left(\varepsilon_{i+1}=\right.$ $\left.\varepsilon_{i}, \delta_{i+1}=\hat{\delta}, i=i+1\right)$

b Model accepted and the largest support so far: design $(i+1)$-th test $\left(\varepsilon_{i+1}=\hat{\varepsilon}, \delta_{i+1}=\hat{\delta}, i=i+1\right)$. Store the current model parameters $\theta$.

Algorithm 2: The structure of R-RANSAC with SPRT.

The algorithm proceeds like standard RANSAC [3, 4], only instead of checking all data points in the model verification step, the data points are evaluated sequentially and hypotheses with low support are rejected early. After a hypothesis is rejected, $\delta$ is re-estimated (Alg. 2, step 2a). Accepted hypotheses are candidates for the RANSAC outcome (see below). The overhead of the evaluation of the likelihood ratio $\lambda_{j}$ eq. (1) is negligible compared to the evaluation of the model versus data point error function.

Probabilities $\varepsilon$ and $\delta$ are different for different data sets and we assume they are unknown. The R-RANSAC with SPRT performs the optimal test derived in Section 2 and uses values of $\varepsilon$ and $\delta$ that are estimated during the sampling process and the test is adjusted to reflect the current estimates.

If the probabilities $\varepsilon$ and $\delta$ are available a-priori, e.g. in some standard setting where the algorithm is run repeatedly, they can be used in the initialization of the algorithm.

Estimation of $\delta$. Since almost all tested models are 'bad' ${ }^{7}$, the probability $\delta$ can be estimated as the average fraction of consistent data points in rejected models. When current estimate $\delta$ differs from the estimate used to design the SPRT (by more than 5\%, for example), new $(i+1)$-th test is designed. The initial estimate $\delta_{0}$ is obtained by geometric considerations, i.e. as a fraction of the area that supports a hypothesised model (a strip around an epipolar line in case of epipolar geometry) to the area of possible appearance of outlier data (the area of the search window). Alternatively, a few models can be evaluated without applying SPRT in order to obtain an initial estimate of $\delta$.

\footnotetext{
${ }^{7}$ RANSAC verifies, on average, $-\log \left(\eta_{0}\right)$ 'good' models, e.g. for the typical $\eta_{0}=0.05$ a 'good' model is hypothesised three times prior to termination of the algorithm.
}

Estimation of $\varepsilon$. In general, it is not possible to obtain an unbiased estimate of $\varepsilon$, since this would require the knowledge of the solution to the optimization problem we are solving. The tightest lower bound on $\varepsilon$ is provided by the size of the largest support so far. It was shown in [6] that a sample with the largest support so far appears $\log k$ times, where $k$ is the number of samples drawn. When such a sample (with support of size $I_{i+1}$ ) appears, new test is designed for $\varepsilon_{i+1}=I_{i+1} / N$. Throughout the course of the algorithm, a series of different tests with $\varepsilon_{0}<\ldots<\varepsilon_{i}<\ldots<\varepsilon$ are performed. The initial value of $\varepsilon_{0}$ can be derived from the maximal time the user is willing to allocate to the algorithm.

The termination criterion. The algorithm is terminated, when the probability $\eta$ of missing a set of inliers larger than the largest support found so far falls under a predefined threshold $\eta_{0}$. In standard RANSAC, where the probability of rejection of a 'good' model is zero, the probability is equal to $\eta_{R}=\left(1-P_{g}\right)^{k}$. In R-RANSAC, the probability of hypothesising and not rejecting a 'good' model is $P_{g}(1-\alpha)$ and the probability $\eta$ becomes as

$$
\eta=\left(1-P_{g}(1-\alpha)\right)^{k} .
$$

In R-RANSAC with SPRT, the SPRT is adjusted to current estimates of $\delta_{i}$ and $\varepsilon_{i}$, so $\alpha$ is no more constant. Theorem 1 , which gives the probability $\alpha$ of rejecting a 'good' model for the test designed for optimal value of $\varepsilon$, does not cover this situation. The following theorem is needed:

Theorem 3 The probability of rejecting a 'good' model with fraction of inliers $\varepsilon$ in a SPRT designed for $\varepsilon_{i}$ and $\delta_{i}$ with threshold $A_{i}$ is

$$
\alpha_{i}=A_{i}^{-h_{i}},
$$

where $h_{i}$ is given by

$$
\varepsilon\left(\frac{\delta_{i}}{\varepsilon_{i}}\right)^{h_{i}}+(1-\varepsilon)\left(\frac{1-\delta_{i}}{1-\varepsilon_{i}}\right)^{h_{i}}=1 .
$$

Proof: For proof see [12, p. 50].

Equation (9) has two solutions, one being $h_{i}=0$. Since $\varepsilon_{i}<\varepsilon, h_{i}>1$ holds for other solution. This solution is found numerically.

Let for each of $l$ tests the following values be stored: the estimated lower bound on the fraction of inliers $\varepsilon_{i}$, the SPRT threshold $A_{i}$, the number of samples $k_{i}$ processed by the test, and $h_{i}$ satisfying (9). Then, the probability $\eta$ is given by

$$
\eta(l)=\prod_{i=0}^{l}\left(1-P_{g}\left(1-A_{i}^{-h_{i}}\right)\right)^{k_{i}} .
$$




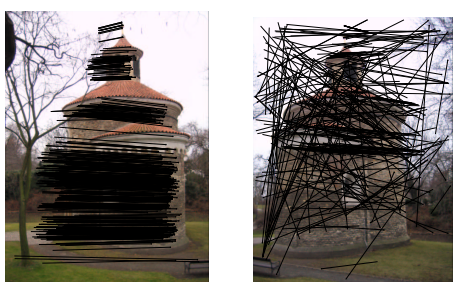

ROTUNDA

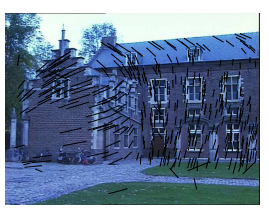

LEUVEN

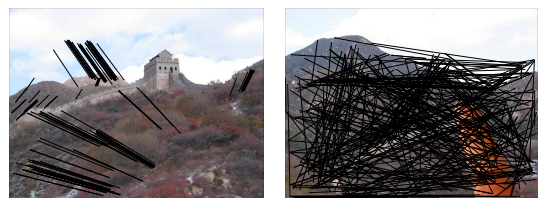

THE GREAT WALL

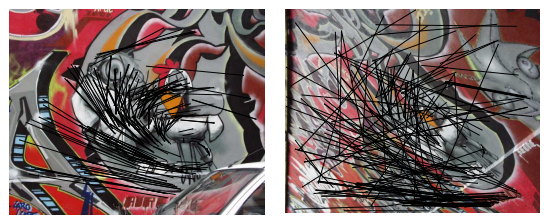

GRAFITTI

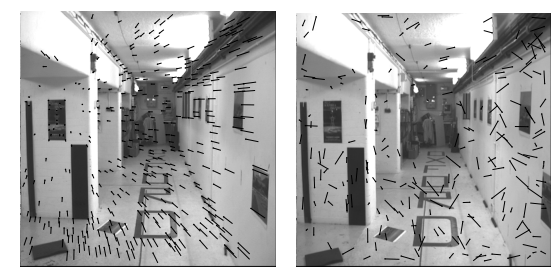

CORRIDOR

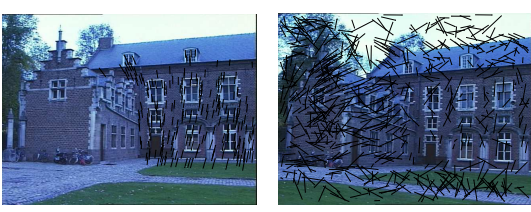

LEUVEN H

Figure 1. The experimental image pairs with inliers (left) and outliers (right) superimposed. Two wide-baseline epipolar geometry experiments ROTUNDA and the GREAT WALL; two narrow-baseline EG experiments LEUVEN and CORRIDOR; two homography experiments GRAFITTI and LEUVEN H.

The number $k_{l}$ of samples that are needed to be drawn with current (i.e. $l$-th) SPRT follows from (10) as

$$
k_{l}=\frac{\log \eta_{0}-\log (\eta(l-1))}{\log \left(1-P_{g} A_{l}^{-1}\right)}
$$

Implementation note: since $\eta>\eta_{R}$ the equation (11) does not have to be evaluated before $\eta_{R}<\eta_{0}$ is satisfied.

\section{Experiments}

Several experiments were performed comparing the proposed R-RANSAC with SPRT with three other RANSAC algorithms: (1) standard RANSAC that verifies all correspondences for every model, (2) R-RANSAC with the $T_{d, d}$ test [6] that rejects the model when the first checked correspondence is not consistent with it $(d=1)$, and (3) R-RANSAC with the a priori SPRT, i.e. the R-RANSAC with SPRT designed for the true values of $\varepsilon$ and $\delta$ (labelled SPRT*). The results achieved with a priori SPRT show the best achievable performance of RANSAC with a randomized verification step for a problem characterized by given $\delta$ and $\varepsilon$.

For epipolar geometry estimation, the time needed to compute model parameters $t_{M}=200$ was set within the range observed in a large number of experiments (i.e. in our implementation, checking whether a correspondence is consistent with a fundamental matrix is 200 times faster than estimating the matrix). The exact value depends on many factors including the CPU speed and type. The constant $\bar{m}_{S}=2.38$ was set to the experimentally observed average of the number of models generated by the 7-point algorithm per sample ${ }^{8}$. The initial value of $\delta$ was set to $\delta_{0}=0.05$. The maximum number of samples was set to 200,000. Since the size of the sample is $m=7$, the $95 \%$ confidence in the solution can be only ensured for $\varepsilon>0.2$, see eq. (7), therefore $\varepsilon_{0}$ was set to $\varepsilon_{0}=0.2$.

For homography estimation $(m=4)$, the values were set as follows $t_{M}=200, \bar{m}_{S}=1, \delta_{0}=0.01, \varepsilon_{0}=0.1$.

The experimental image pairs are displayed in Fig. 1. The number $N$ of correspondences as well as the true values of $\varepsilon$ and $\delta$ estimated by evaluation 100,000 verifications of random models are summarized in Tab. 2 . The results of compared algorithms are shown in Table 1.

As a consequence of the randomization of model verification that erroneously rejects some 'good' models, on average, the randomized algorithms must draw a larger number of samples than standard RANSAC. This is confirmed in the first column of Table 1. This small increase is more than compensated by the reduction in the number of data points (correspondences) checked on average per model. The running time of RANSAC is reduced by factors ranging from 2.8 to 10.9 In all experiments the SPRT outperforms the $T_{d, d}$ test.

\section{Conclusions}

We have derived an optimal sequential strategy for randomized evaluation of model quality in RANSAC. A method for estimating the two probabilities characterizing the prob-

\footnotetext{
${ }^{8}$ It is known that the 7-point algorithm produces 1 to 3 potential models. In experiments, the average number of models per sample -2.38 - has been observed consistently in a number of scenes. No theoretical justification of the stability of this average is known to the authors.
} 


\begin{tabular}{|c|c|c|c|c|c|}
\hline \multicolumn{6}{|c|}{ The ROTUNDA A experiment } \\
\hline & $k$ & models & $\mathrm{vpm}$ & time & spd-up \\
\hline RANSAC & 195 & 470 & 886.0 & 4.3 & 1.0 \\
\hline$T_{d, d}$ & 345 & 834 & 49.0 & 1.4 & 3.1 \\
\hline SPRT $^{*}$ & 201 & 486 & 29.1 & 0.8 & 5.4 \\
\hline SPRT & 200 & 483 & 41.5 & 0.9 & 4.9 \\
\hline \multicolumn{6}{|c|}{ The GREAT WALL experiment } \\
\hline & \multirow{2}{*}{$\begin{array}{c}k \\
48085\end{array}$} & models & vpm & \multirow{2}{*}{\begin{tabular}{|r|r|} 
time \\
667
\end{tabular}} & \begin{tabular}{l|l} 
e & spd-up
\end{tabular} \\
\hline RANSAC & & \multirow{2}{*}{$\begin{array}{l}122223 \\
571059\end{array}$} & 507. & & 1.0 \\
\hline$T_{d, d}$ & 224865 & & \multicolumn{2}{|c|}{6.9} & 1.0 \\
\hline SPRT $^{*}$ & 56678 & 143993 & 14.4 & 178 & 3.8 \\
\hline SPRT & 5579 & & \begin{tabular}{l|l}
1 & 16.
\end{tabular} & 17 & 3.7 \\
\hline \multicolumn{6}{|c|}{ The CORRIDOR experiment } \\
\hline & $k$ & models & $\mathrm{vpm}$ & time & spd-up \\
\hline RANSAC & 145 & 344 & 600.0 & 2.3 & 1.0 \\
\hline$T_{d, d}$ & 243 & 576 & 86.6 & 1.2 & 2.0 \\
\hline SPRT $^{*}$ & 154 & 366 & 32.9 & 0.6 & 3.7 \\
\hline SPRT & 153 & 364 & 77.2 & 0.8 & 2.8 \\
\hline \multicolumn{6}{|c|}{ The LEUVEN experiment } \\
\hline & $k$ & models & vpm & time & spd-up \\
\hline RANSAC & 1855 & 4434 & 786.0 & 35.5 & 1.0 \\
\hline$T_{d, d}$ & 4490 & 10730 & 27.5 & 14.8 & 2.4 \\
\hline SPRT* $^{*}$ & 2025 & 4839 & 20.4 & 6.8 & 5.3 \\
\hline SPRT & 1982 & 4736 & 23.2 & 6.8 & 5.2 \\
\hline \multicolumn{6}{|c|}{ The GRAFFITI experiment } \\
\hline & $k$ & models & vpm & time & spd-up \\
\hline RANSAC & 121 & 121 & 405.0 & 3.0 & 1.0 \\
\hline$T_{d, d}$ & 287 & 287 & 16.7 & 1.0 & 2.8 \\
\hline SPRT $^{*}$ & 132 & 132 & 35.1 & 0.6 & 4.6 \\
\hline SPRT & 129 & 129 & 47.1 & 0.7 & 4.1 \\
\hline \multicolumn{6}{|c|}{ The homography LEUVEN experiment } \\
\hline & $k$ & models & vpm & time & spd-up \\
\hline RANSAC & 1203 & 1203 & 789.0 & 53.1 & 1.0 \\
\hline$T_{d, d}$ & 5323 & 5323 & 3.4 & 15.0 & 3.5 \\
\hline SPRT $^{*}$ & 1351 & 1351 & 14.5 & 4.7 & 11.2 \\
\hline SPRT & 1266 & 1266 & 20.7 & 4.9 & 10.9 \\
\hline
\end{tabular}

Table 1. The comparison of RANSAC, R-RANSAC with $T_{d, d}$ test, a priori SPRT* and SPRT: the number of samples $(k)$, the number of models (models), the number of checked correspondences per model (vpm), time in ms (time), and relative speed-up with respect to standard RANSAC (spd-up). Averaged over 500 runs.

lem and critically influencing the design of the optimal strategy was proposed. A termination criterion maintaining the $1-\eta$ confidence in the solution was derived.

Properties of R-RANSAC with SPRT were tested on wide range of standard data. Tests included epipolar geometry estimation in both wide baseline setting and narrow

\begin{tabular}{|c|c|c|c|}
\hline & corr & $\varepsilon$ & $\delta$ \\
\hline ROTUNDA & 893 & 0.60 & 0.052 \\
\hline THE GREAT WALL & 514 & 0.28 & 0.014 \\
\hline CORRIDOR & 607 & 0.56 & 0.142 \\
\hline LEUVEN & 793 & 0.47 & 0.032 \\
\hline GRAFFITI & 409 & 0.51 & 0.018 \\
\hline LEUVEN H & 793 & 0.29 & 0.004 \\
\hline
\end{tabular}

Table 2. The number of correspondences (corr), fraction of inliers $(\varepsilon)$, the probability of a correspondence being consistent with bad model $(\delta)$.

baseline settings and homography estimation. The method was 2.8 to 10 times faster than RANSAC and up to 4 times faster than R-RANSAC with $T_{d, d}$ test.

\section{References}

[1] O. Chum, J. Matas, and J. Kittler. Locally optimized RANSAC. In Proc. DAGM. Springer-Verlag, 2003.

[2] Ondřej Chum. Two-view geometry estimation by random sample and consensus. PhD thesis, CMP, CTU in Prague, 2005.

[3] M.A. Fischler and R.C. Bolles. Random sample consensus: A paradigm for model fitting with applications to image analysis and automated cartography. CACM, 24(6):381-395, June 1981.

[4] Richard Hartley and Andrew Zisserman. Multiple view geometry in computer vision. Cambridge University, Cambridge, 2nd edition, 2003.

[5] Peter M. Lee. Sequential probability ratio test. University of York. www.york.ac.uk/depts/maths/teaching/pml/ais/sprt.ps.

[6] Jiří Matas and Ondřej Chum. Randomized RANSAC with $T_{d, d}$ test. Image and Vision Computing, 22(10):837-842, September 2004.

[7] D.R. Myatt, P.H.S. Torr, S.J. Nasuto, J.M. Bishop, and R. Craddock. Napsac: High noise, high dimensional robust estimation - it's in the bag. In $B M V C O 2$, volume 2, pages 458-467, 2002.

[8] D. Nister. Preemptive RANSAC for live structure and motion estimation. In Proc. ICCV03, volume I, pages 199-206, October 2003.

[9] B. Tordoff and D.W. Murray. Guided sampling and consensus for motion estimation. In Proc. 7th ECCV, volume 1, pages 82-96. Springer-Verlag, 2002.

[10] P. H. S. Torr and A. Zisserman. MLESAC: A new robust estimator with application to estimating image geometry. CVIU, 78:138-156, 2000.

[11] P.H.S. Torr, A. Zisserman, and S.J. Maybank. Robust detection of degenerate configurations while estimating the fundamental matrix. CVIU, 71(3):312-333, Sep. 1998.

[12] Abraham Wald. Sequential analysis. Dover, New York, 1947. 